\title{
Increasing task demand by obstructing object recognition increases boundary extension
}

\author{
Ralph G. Hale ${ }^{1}$ James M. Brown ${ }^{1} \cdot$ Benjamin A. McDunn ${ }^{1}$
}

Published online: 7 March 2016

(C) Psychonomic Society, Inc. 2016

\begin{abstract}
Individuals consistently remember seeing widerangle versions of previously viewed scenes than actually existed. The multi-source model of boundary extension (BE) suggests many sources of information contribute to this visual memory error. Color diagnosticity is known to affect object recognition with poorer recognition for atypically versus typically colored objects. Scenes with low-color diagnostic main objects and two versions of scenes with high-color diagnostic main objects (typically and atypically colored) were tested to determine if the reduced ability to identify the main object in a scene influences BE. Scenes were presented to one group of participants for $46 \mathrm{~ms}$ and another group for $250 \mathrm{~ms}$. Each scene was followed by a mask and a request for a recognition response concerning the identity of the main object. The scene was then immediately presented again for testing and participants rated it as depicting a more close-up view, more wideangle, or the same view as before. The study demonstrates that poorer encoding of main objects in scenes leads to increased $\mathrm{BE}$, but trial-by-trial recognition accuracy had no relationship to $\mathrm{BE}$ magnitude. This finding provides further insight into the impact of task demand and main object recognition on BE.
\end{abstract}

Keywords Boundary extension $\cdot$ Scene memory $\cdot$ Color diagnosticity

Boundary extension (BE) is an error of commission in which observers confidently remember more information from a scene than was present in the studied view (Intraub \&

Ralph G. Hale

rusty7@uga.edu

1 University of Georgia, Athens, GA 30602, USA
Richardson, 1989). Functionally, BE is likely a useful phenomenon for the observer in predicting outlying or occluded visual information. Utilizing current visual information in conjunction with extrapolation of what potentially lies beyond the view boundaries allows for prediction of visual information prior to its presence on the retina, helping with tasks like navigation. Intraub's $(2010,2012)$ multisource model of scene perception suggests that $\mathrm{BE}$ arises due to a combination of information from such sources as the visual input, semantic information, amodal perception at the view boundaries (Hale, Brown, McDunn, \& Siddiqui, 2014), and associations between the main objects and the depicted scene. Recent research indicates that semantic information may not be necessary for BE to occur. For example, BE is still present when semantic information is reduced by using "scenes" of monochromatic abstract shapes on random dot backgrounds (Hale et al., 2014; McDunn, Siddiqui, \& Brown, 2014).

Although the recent findings of Hale et al. (2014) and McDunn et al. (2014) suggest that semantic information is not necessary for $\mathrm{BE}$ to occur, the ability to recognize a main object in a scene may still play a role in BE magnitude. This could be tested by manipulating the ability to identify the main object in a scene. Comparing a condition in which recognition of the main object is difficult to one in which semantic information was fully available to the observer (i.e., main object recognition was easier) could shed further light on the role of main object recognition in $\mathrm{BE}$.

\section{Manipulating object recognition}

The speed by which someone can recognize an object or scene can be aided or impeded depending on the method of presentation. There are many scenarios that could affect recognition accuracy. For instance, simply rotating an image of a human 
face $180^{\circ}$ can drastically decrease recognition accuracy (Van Belle, Graef, Verfaillie, Rossion, \& Lefevre, 2010). One way to aid object recognition is to use high-color diagnostic (HCD) images that are typically colored (Tanaka \& Presnell, 1999). Color diagnosticity is the level to which an object or scene is identified by its constituent colors (Tanaka, Weiskopf, \& Williams, 2001). HCD objects or scenes contain colors with which they are commonly associated. For example, all drivers should know that brake lights, stop lights, and stop signs are red. When a driver perceives the color red ahead of him or her, that driver is likely to slow down and prepare to stop. This realization could occur moments before he or she precisely identifies the perceived "red" in his or her field of view. Another example of HCD objects can be imagined in the context of shopping for fruit at the grocery store. Bananas can be found with ease by doing a quick visual scan of the produce section searching for the canonical bright yellow hue commonly associated with them.

The "color advantage" seen in these examples could potentially become a "color disadvantage" if atypically colored versions of these HCD images are used. In other words, replacing the canonical colors for a particular HCD object or scene with unlikely or incorrect colors (e.g., a purple banana) can actually impede or reduce the speed of recognition (Nagai \& Yokosawa, 2003; Tanaka \& Presnell, 1999). Using the previous HCD real world examples, a stop sign that has faded to silver and a banana that is green or brown could initially cause a slight "color disadvantage" due to the discrepancy between color expectation and reality. This conflict would soon be resolved by a combination of top-down and bottom-up processes allowing object recognition to occur despite the unusual coloration. If altering the canonical colors of HCD objects or scenes can temporarily inhibit or interfere with recognition abilities, then typically colored versions are likely to be recognized more quickly than atypically colored versions (e.g., Bramao, Faisca, Petersson, \& Reis, 2010).

This increased difficulty in recognizing objects due to color diagnosticity is a potentially useful tool in studying the role of main object recognition in BE. Scenes containing typically rather than atypically colored HCD objects are identified more quickly, suggesting that semantic information is available sooner and more readily in these scenes (Bramao et al., 2010). By comparison, low-color diagnostic (LCD) objects should not differ significantly in recognition accuracy from typically colored HCD objects. For example, a balloon can be virtually any color imaginable and therefore has very low color diagnosticity. LCD objects should be recognized just as quickly as typically colored HCD objects because constituent colors of LCD objects provide little or no advantage or disadvantage (e.g., Nagai \& Yokosawa, 2003). We constructed a $\mathrm{BE}$ paradigm implementing this method of decreasing main object identification ability.
From the perspective of Intraub's multi-source model, a less precise initial encoding should lead to greater $\mathrm{BE}$ (Intraub, Daniels, Horowitz, \& Wolfe, 2008). Intraub and colleagues (2008) found that BE was greater when trials had an additional task (e.g., search task) leading to a less precise initial encoding. A poorer memory representation will increase the likelihood of a source monitoring error because of difficulty differentiating the memory of the actual scene viewed at study from the extrapolated information created during perception. Reducing main object identification ability by using atypically colored HCD objects versus typically colored HCD or LCD objects should also lead to a less precise initial encoding of the scene. A recognition deficit is likely to exist for atypically colored HCD objects displayed for a brief duration (e.g., $46 \mathrm{~ms}$ ). This condition has the highest task demand and should therefore produce the greatest BE (e.g., Intraub et al., 2008). At a longer stimulus duration (e.g., 250 $\mathrm{ms}$ ), information needed to positively identify the main object in a scene should be more available for all three picture types leading to higher identification accuracy and similar BE ratings (i.e., poorer encoding limited to the shorter stimulus duration). If access to information needed to positively identify the main object in a scene is diminished (as demonstrated in our identification pilot experiment), then BE should be greater at $46 \mathrm{~ms}$ duration for scenes with an atypically colored HCD main object due to the poorer quality of information stored and the increased reliance on extrapolation in order to recall the studied view.

\section{Method}

\section{Participants}

Participants were 70 undergraduate students from the University of Georgia research pool (43 female, 27 male; mean age $=19.6$ years). All participants had normal or corrected-to-normal vision, normal color vision (verified by pseudoisochromatic plates), no history of an attention deficit disorder, no history of epilepsy, and a dominant right hand (i.e., "right-handed"). Participants were at least 18 years old at the time of the experiment. All research was conducted in accordance with the Declaration of Helsinki and under the approval of University of Georgia Institutional Review Board (IRB) ethical guidelines for research involving human participants. Participants received partial course credit as compensation for their participation.

\section{Stimuli \& apparatus}

To select our stimuli, we trained a separate group of participants $(n=10)$ to categorize objects based on color diagnosticity by giving precise definitions of color 
diagnosticity, providing an example of a HCD object (i.e., a Smurf) and a LCD object (i.e., a balloon) not from the potential stimulus set, and answering any questions the participants had regarding color diagnosticity. The participants ranked objects for color diagnosticity using a Likert-type response from 0 to 10 where 0 was very LCD and 10 was very HCD. Objects with an average ranking of $0-2$ or 8-10 defined our LCD and HCD object stimuli, respectively. Any object that did not fall into one of these ranges was excluded from the study.

Using these ratings as inclusion criteria, we randomly selected 20 images of scenes with HCD central objects (i.e., half typically colored and half atypically colored) and 10 images of scenes with LCD central objects to serve as stimuli in the experiment. To create atypically colored objects, typically colored objects were modified in Adobe Photoshop CS6 by adjusting hue and color balance (see Fig. 2). For atypically colored HCD objects, approximately $65 \%$ were complimentary or near complimentary color versions of the original typically colored HCD objects. The remaining objects were changed to a color that was "most atypical" from the original color if the complimentary color did not seem atypical for that object. Two color noise masks were created using Paint, each consisting of overlapping blocks filled with 1 of 12 solid colors. Each color noise mask contained black, white, grly, red, orange, yellow, dark green, light green, dark blue, light blue, and purple in an approximately equal and random distribution on the screen. These colors were representative of the large range of colors present in all stimulus picture types. The second mask was a $180^{\circ}$ rotated version of the first mask to further decrease the likelihood of any afterimage effects due to the location of a particular color within a stimulus. All stimuli (i.e., scenes) had an $800 \times 600$ pixel resolution that encompassed the entire screen and were displayed for either $46 \mathrm{~ms}$ or $250 \mathrm{~ms}$. Images were presented on a monitor operating at an $85-\mathrm{Hz}$ refresh rate using E-Prime v.2 software. Participants viewed the monitor from $181.4 \mathrm{~cm}$ creating a

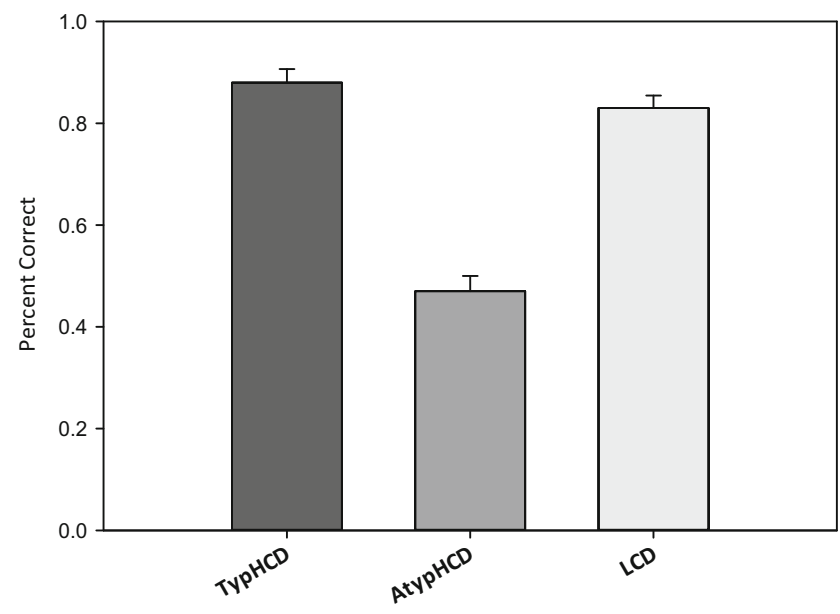

Fig. 1 Pilot identification task results for typically colored HCD, atypically colored $\mathrm{HCD}$, and LCD objects at $46 \mathrm{~ms}$ duration

visual angle of $8^{\circ}$ (height) $\times 11.1^{\circ}$ (width). Overhead lights remained on for the duration of the experiment. Responses were recorded using the computer keyboard. A chin rest was used to minimalize head movements and fix gaze distance during each trial.

\section{Procedure}

To ensure our stimuli would impact recognition accuracy as expected, a pilot experiment was conducted in which a separate 15 participants were shown a series of 30 scenes (i.e., those selected in the first pilot experiment) on a computer monitor for $46 \mathrm{~ms}$ each, followed by a colored noise mask for $200 \mathrm{~ms}$. Participants were then asked to type an identification response naming the object. Each stimulus contained a central object that was typically colored HCD, atypically colored HCD, or LCD (i.e., 10 of each picture type). As expected, identification accuracy of atypically colored HCD objects was
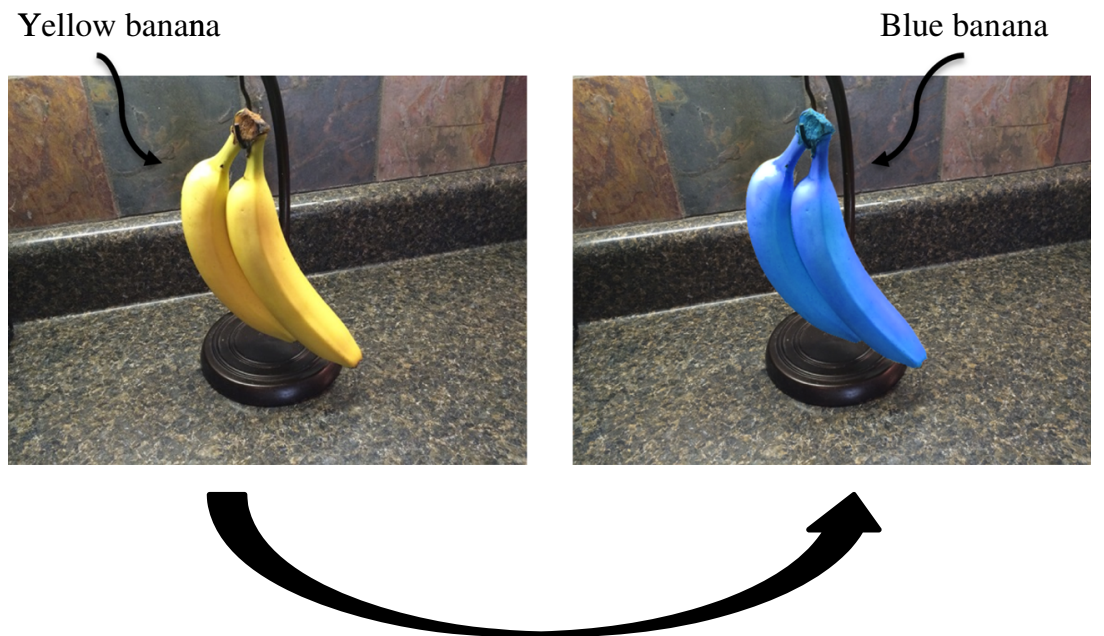

Fig. 2 Example of a HCD object that has been transformed into an atypically colored HCD object 
inferior to typically colored HCD objects $\left(t_{14}=3.21, p<.01\right)$ and LCD objects $\left(t_{14}=-2.93, p<.05\right.$; see Fig. 1$)$ with no difference between typically colored HCD and LCD objects.

The main experiment was a $2 \times 3$ mixed design with scene display duration manipulated between subjects $(n=35$ for both 46 and $250 \mathrm{~ms}$ ) and picture type manipulated within subjects (typically colored HCD, atypically colored HCD, and LCD). Participants were read the instructions by the experimenter as they were displayed on the computer monitor. Participants were given one practice trial followed by 30 experimental trials. Each trial consisted of 7 parts: (1) a fixation stimulus displayed until the participant began the trial by pressing the spacebar, (2) a scene randomly selected from one of the three picture types and displayed for either 46 or $250 \mathrm{~ms}$, (3) two color noise masks displayed for $100 \mathrm{~ms}$ each, (4) an object identification response prompt for this stimulus which was entered using the keyboard, (5) a confidence rating of the participant's identification response, (6) the same test stimulus with a BE rating response prompt, and (7) a confidence response prompt for this BE rating (see Fig. 3). Object identification accuracy was determined by having the participant type the name of the central main object of the scene. Responses were classified as correct or incorrect based on whether or not the response indicated any level of recognition of the object. The BE rating was given on a 5-point Likerttype scale: (1) much closer up, object looks much bigger; (2) slightly closer-up, object looks slightly bigger; (3) the same; (4) slightly wider angle, object looks slightly smaller; (5) much more wide-angle, object looks much smaller. BE ratings were later adjusted from a range of 1 to 5 to a range of -2 to +2 for the purposes of analysis. For both confidence level ratings, participants were asked "How sure are you about your

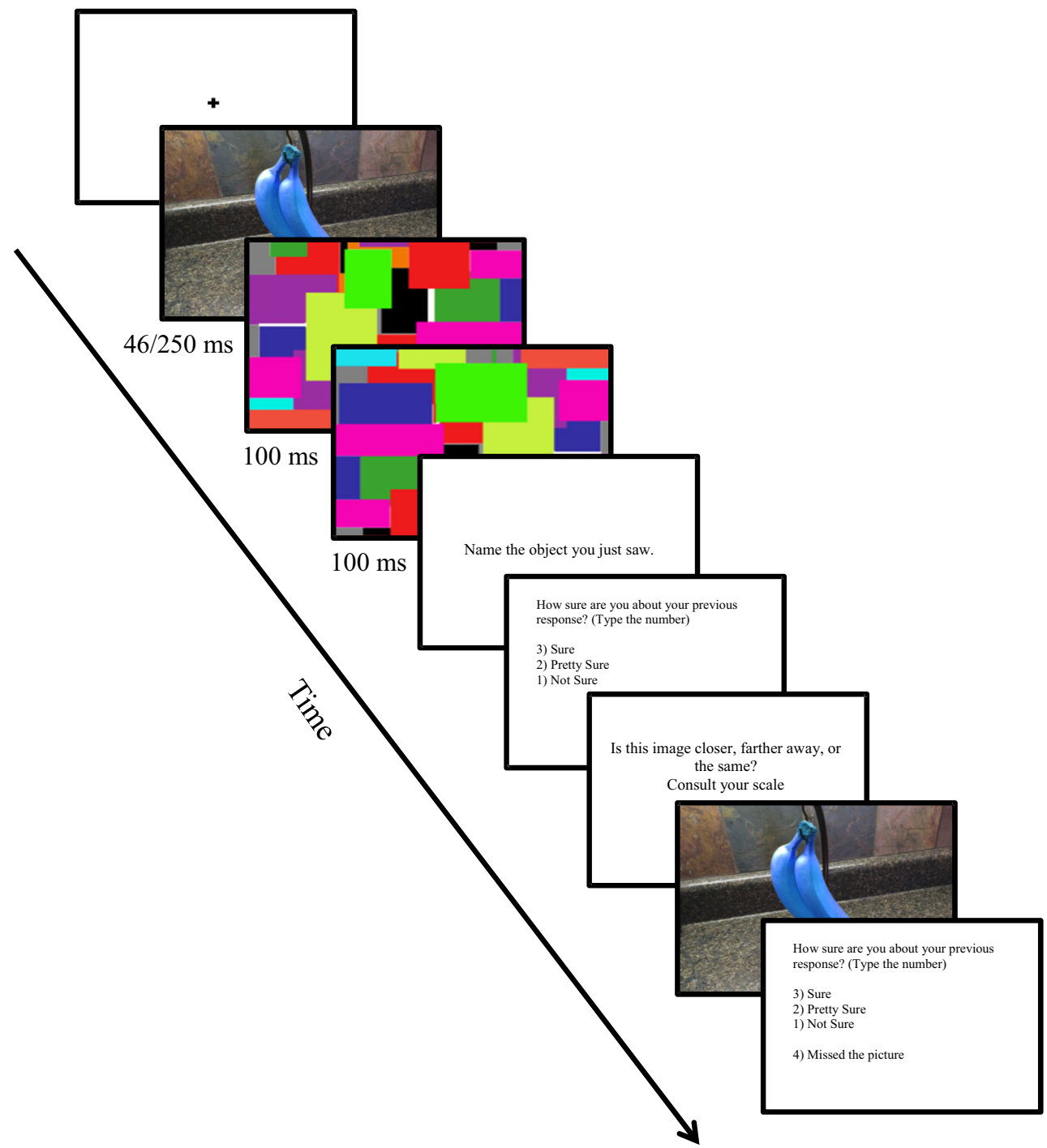

Fig. 3 Individual trial breakdown over time. Components without a stimulus duration caption were terminated by participant response. Original stimulus was shown for either 46 or $250 \mathrm{~ms}$ depending on condition. Color noise mask was shown for $100 \mathrm{~ms}$ followed by a $180^{\circ}$ rotated version of this mask for $100 \mathrm{~ms}$. The BE rating scale was provided on a separate sheet of paper for the participant to refer to each trial. The confidence rating scale was presented on screen simultaneously with the confidence prompt 
Table 1 Identification values for both stimulus durations in all three picture types (i.e., typically colored HCD, atypically colored HCD, and LCD). Values represent percent correct $(1.0=100 \%$ correct $)$ based on individual means

\begin{tabular}{llllll}
\hline & 46 ms duration & & \multicolumn{2}{c}{250 ms duration } \\
\hline Picture type & HCD & HCD & LCD & HCD & HCD \\
& typical & atypical & & typical & atypical \\
Identification percentage & .84 & .59 & .88 & .90 & .79 \\
\hline
\end{tabular}

previous response?" and rated their confidence on a 4-point Likert-type scale: (3) sure, (2) pretty sure, (1) not sure, and (4) missed the picture. Trials in which a confidence rating of 4 was given for the participant's BE rating were removed from the analyses $(3.71 \%$ of trials at $46 \mathrm{~ms}$ and $0.57 \%$ of trials at $250 \mathrm{~ms}$; see Table 3). The confidence rating for the object identification accuracy task was not used in the analyses. There was concern that participants were convoluting a response of 4 on the confidence report with a response that they could not identify the main object. We expected participants to be less confident in their identification ratings for atypically colored HCD objects at $46 \mathrm{~ms}$ due to the difficulty of this task. Removing trials in which participants responded with a confidence of 4 would then artificially inflate the accuracy rate, especially in this condition (see Table 3 ).

\section{Results}

A $2 \times 3$ mixed ANOVA with stimulus duration (46 and $250 \mathrm{~ms}$ ) as the between subjects factor and picture type (typically colored HCD, atypically colored HCD, and LCD) as the within subjects factor was conducted for analysis of object identification accuracy. After applying a Greenhouse-Geisser correction for sphericity violation, the ANOVA showed a significant interaction between picture type and stimulus duration, $F_{1.76,119.45}=32.75, p<$ .05 , partial $\eta^{2}=.325$, and also a significant main effect of picture type, $F_{1.76,119.45}=276.32, p<.05$, partial $\eta^{2}=$ .803. The main effect of stimulus duration was also significant, $F_{1,68}=33.14, p<.05$, partial $\eta^{2}=.328$. Levene's test of equality of error variances showed this assumption to be violated for the atypically colored HCD condition; however, a more robust Brown-Forsythe test yielded the same result. The main effect of duration indicates that participants were better at object identification for the 250-ms condition compared to the 46-ms condition. The poor recognition at $46 \mathrm{~ms}$ replicates the results of our pilot experiment. As can be seen in Fig. 4, the picture type with the largest mean difference in accuracy between 46 and $250 \mathrm{~ms}$ was the atypically colored HCD images. Other picture types were near ceiling in accuracy for both durations. All accuracy values are reported in Table 1.

A planned comparison $t$ test was calculated for each condition's BE ratings to determine if they differed significantly from zero (i.e., a rating significantly less than zero signifying the presence of $\mathrm{BE}$ ). A Bonferroni correction was used to adjust our $p$ value from $p<.05$ to $\mathrm{p}<0.0083$ for these additional six comparisons. In all three picture types for 46 and $250 \mathrm{~ms}$, average BE values were significantly less than zero (typically colored HCD at $46 \mathrm{~ms}, t_{34}=-5.551, p<0.0083$; atypically colored HCD at $46 \mathrm{~ms}, t 34=-9.865, p<0.0083$; LCD at $46 \mathrm{~ms}, t 34=-5.022, p<0.0083$; typically colored at $250 \mathrm{~ms}, t_{34}=-5.662, p<0.0083$; atypically colored HCD at $250 \mathrm{~ms}, t_{34}=-6.275, p<0.0083 ; \mathrm{LCD}$ at $250 \mathrm{~ms}, t_{34}=-$ $6.735, p<0.0083$ ) demonstrating that $\mathrm{BE}$ was present in every condition.

Having established the presence of BE across all conditions, we next tested if diminished recognition of the main object increased BE magnitude. A repeated measures ANOVA of the 46-ms condition showed a significant effect of picture type on $\mathrm{BE}$ ratings, $F_{2,68}=6.457, p<.05$, with a moderately strong effect size, partial $\eta^{2}=.160$. Pairwise comparisons using a Bonferroni correction showed a significant difference between $\mathrm{BE}$ ratings for atypically colored $\mathrm{HCD}$ objects and the other two picture types, but no significant difference between typically colored HCD objects and LCD

Table 2 BE values for both stimulus durations in all three picture types (i.e., typically colored HCD, atypically colored HCD, and LCD). A score of " 0 " indicates that the recalled image from study and the viewed image at test are identical

\begin{tabular}{|c|c|c|c|c|c|c|}
\hline \multirow[t]{2}{*}{ Picture type } & \multicolumn{3}{|c|}{$46 \mathrm{~ms}$ duration } & \multicolumn{3}{|c|}{$250 \mathrm{~ms}$ duration } \\
\hline & $\begin{array}{l}\mathrm{HCD} \\
\text { typical }\end{array}$ & $\begin{array}{l}\mathrm{HCD} \\
\text { atypical }\end{array}$ & LCD & $\begin{array}{l}\mathrm{HCD} \\
\text { typical }\end{array}$ & $\begin{array}{l}\mathrm{HCD} \\
\text { atypical }\end{array}$ & LCD \\
\hline Boundary rating & -0.4086 & -0.6314 & -0.3429 & -0.3914 & -0.4114 & -0.3486 \\
\hline
\end{tabular}


Table 3 Confidence rating percentages for both stimulus durations in all three picture types (i.e., typically colored HCD, atypically colored HCD, and LCD). Participants were asked "How sure are you about your previous response?" and rated their confidence on a 4-point Likert-type scale: (3) sure, (2) pretty sure, (1) not sure, and (4) missed the picture

\begin{tabular}{|c|c|c|c|c|c|c|c|c|c|}
\hline \multirow[t]{2}{*}{ Duration } & \multirow[t]{2}{*}{ Rating } & \multicolumn{4}{|l|}{ ID } & \multicolumn{4}{|l|}{$\mathrm{BE}$} \\
\hline & & Atyp & Typ & $\mathrm{LCD}$ & Total & Atyp & Typ & $\mathrm{LCD}$ & Total \\
\hline \multirow[t]{4}{*}{$46 \mathrm{~ms}$} & 3 & 26.29 & 33.14 & 32.86 & 30.76 & 22.00 & 33.43 & 32.57 & 29.33 \\
\hline & 2 & 30.57 & 35.71 & 35.14 & 33.81 & 33.14 & 35.71 & 37.43 & 35.43 \\
\hline & 1 & 30.57 & 28.00 & 27.14 & 28.57 & 38.00 & 28.57 & 28.00 & 31.52 \\
\hline & 4 & 12.57 & 3.14 & 4.86 & 6.86 & 6.86 & 2.29 & 2.00 & 3.71 \\
\hline \multirow[t]{4}{*}{$250 \mathrm{~ms}$} & 3 & 70.57 & 78.29 & 80.57 & 76.48 & 34.29 & 46.57 & 39.71 & 40.19 \\
\hline & 2 & 7.43 & 10.29 & 10.29 & 9.33 & 48.57 & 41.14 & 50.00 & 46.57 \\
\hline & 1 & 19.71 & 19.57 & 8.29 & 12.86 & 16.00 & 12.00 & 10.00 & 12.67 \\
\hline & 4 & 2.29 & 0.86 & 0.86 & 1.33 & 1.14 & 0.29 & 0.29 & 0.57 \\
\hline
\end{tabular}

objects was found. As hypothesized, the condition expected to lead to the poorest encoding of the study stimuli also yielded the greatest BE (see Fig. 5). A repeated measures ANOVA of the 250-ms condition showed the effect of picture type on $\mathrm{BE}$ ratings was not significant, $F_{2,68}=0.304, p>.05$, partial $\eta^{2}=$ .009 . This result indicates that the additional study time in the 250-ms condition resulted in BE ratings converging to similar values, regardless of picture type. Once participants had time to overcome the atypical colors of these objects, there was no difference in BE. All BE values are reported in Table 2.

These analyses show that diminished main object recognition increased BE in our experiment; however, the means by which this effect took place could still be one of two possibilities. First, it is possible that BE magnitude for a particular picture is directly influenced by whether or not recognition for the main object in the picture is successful. In this case, it is expected that trials in which main object recognition is successful will show less BE than trials in which main object

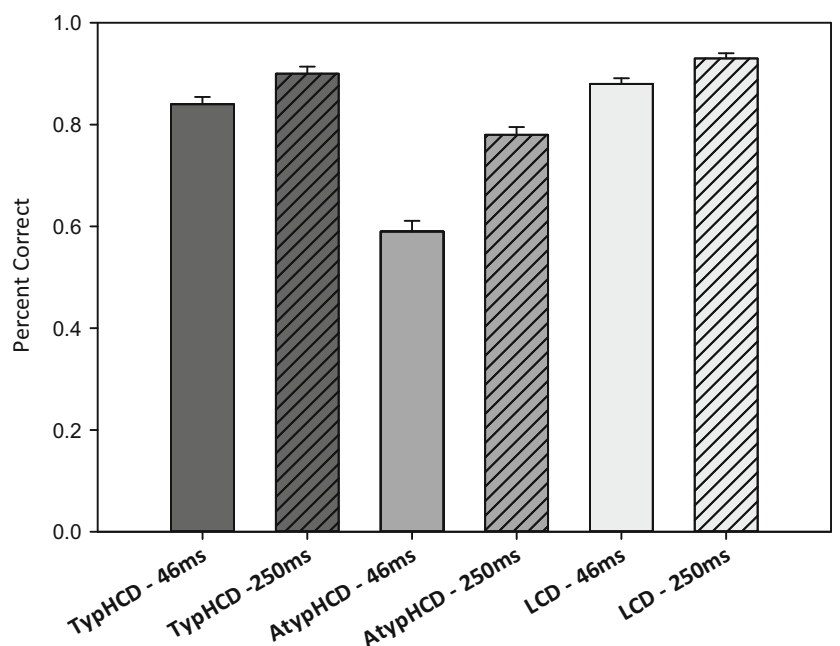

Fig. 4 Identification task results for typically colored HCD, atypically colored HCD, and LCD objects at 46 (solid bars) and $250 \mathrm{~ms}$ (striped bars) stimulus durations recognition is unsuccessful. A second possibility is that the atypically colored HCD objects in the 46-ms condition had increased $\mathrm{BE}$ due to the greater difficulty of the recognition task in this condition, regardless of whether or not object recognition was successful. In this case, the effect main object recognition has on $\mathrm{BE}$ is mediated by the general increase in difficulty of the recognition task. This second possibility is more similar to the increased $\mathrm{BE}$ due to the divided attention effect observed by Intraub and colleagues (2008) when a search task was used alongside a BE task to create a less precise initial encoding.

A trial-by-trial analysis was conducted to examine the relationship between identification accuracy (correct or incorrect) and BE ratings at $46 \mathrm{~ms}$ for atypical stimuli in order to test the possibility that recognition of the main object directly impacted $\mathrm{BE}$ ratings. A point biserial correlation found $\mathrm{BE}$ ratings were not related to identification accuracy for trials in this condition $r_{342}=-.029, p>.05$, suggesting that increased

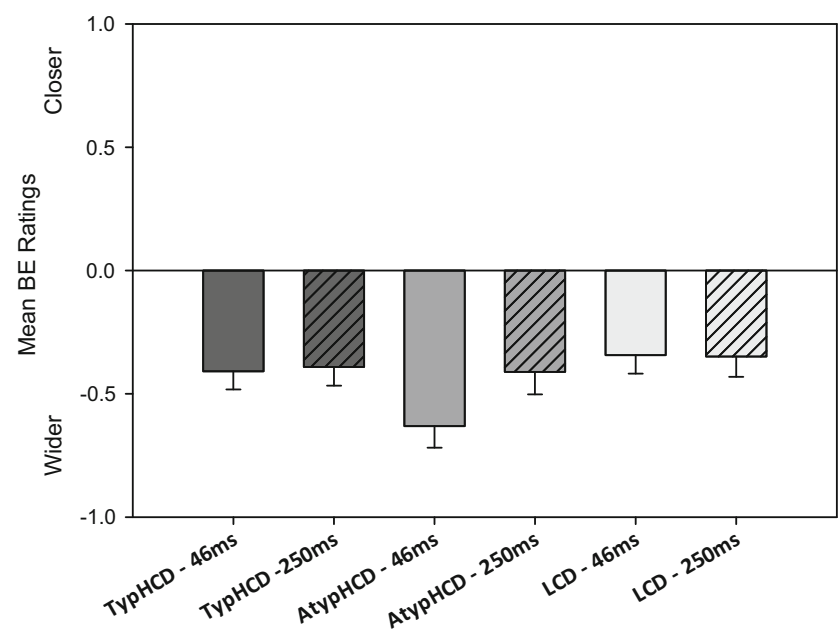

Fig. 5 BE ratings for typically colored HCD, atypically colored HCD, and LCD objects at 46 (solid bars) and $250 \mathrm{~ms}$ (striped bars) stimulus durations 
task demand was likely the cause of the increase in BE rather than a direct relationship between object identification and BE.

\section{Discussion}

When individuals view a scene, they consistently recall seeing more information than was actually present in the original exposure. The magnitude of BE can be larger or smaller depending on the circumstances. In the instance of a degraded memory representation due to poorer initial encoding, information is sparsely available leading to greater reliance on extrapolation. Our participants could correctly identify typically colored HCD objects at a higher accuracy than atypically colored versions at the shorter (i.e., $46 \mathrm{~ms}$ ) viewing duration. The peculiarity of the color information in the atypically colored stimuli interfered with the availability of information required for main object recognition enough to significantly affect recognition accuracy and encoding in visual memory. This poorer initial encoding resulted in greater BE for these scenes than for typically colored or LCD versions. At the longer (i.e., $250 \mathrm{~ms}$ ) viewing duration, this atypical color disadvantage observed at the shorter duration for HCD objects was greatly reduced. While BE was present for all picture types and stimulus durations, no difference in BE ratings between picture types was found at the longer viewing duration because participants could readily identify objects in all picture types.

Our original hypothesis stated that a reduction in identification ability would cause an increase in BE. Our data show that this appears to be the case, with a reduction in identification ability and an increase in BE for the atypically colored stimuli at $46 \mathrm{~ms}$ as predicted. However, rather than a direct connection between identification ability and $\mathrm{BE}$ magnitude, identification ability and $\mathrm{BE}$ ratings are mediated by the increased difficulty of the task. This supports Intraub and colleagues' (2008) finding that increased task demand causing a poor initial encoding can increase the magnitude of BE. The peculiarity of the atypical stimuli combined with the already difficult task of attempting to identify an image and store it for recall with just $46 \mathrm{~ms}$ of stimulus duration caused this particular condition to have greater $\mathrm{BE}$ than any of the other conditions. At $250 \mathrm{~ms}$, recognition task difficulty was equal for all pictures types leading to comparable BE for all stimuli. By manipulating color typicality and viewing duration of $\mathrm{HCD}$ objects, we created a scenario (i.e., atypically colored HCD objects at the short stimulus duration) by which only a degraded version of the image could be encoded. Under these circumstances, participants remembered a more extrapolative, wider-angle version at test.
Although diminished recognition of main objects in our experiment increased $\mathrm{BE}$, the trial-by-trial analysis seems to support previous research suggesting that semantic information may not be critical for BE to occur (Hale et al., 2014; McDunn et al., 2014). Successful or unsuccessful recognition had no relationship with $\mathrm{BE}$ ratings, as shown by the weak correlation reported above. This finding also supports the importance of the quality of initial memory encoding to the magnitude of the BE error found by Intraub and colleagues (2008) using a completely different manipulation. The greater BE found for atypical HCD trials at $46 \mathrm{~ms}$ was most likely a result of the general increase in cognitive demand created by the difficulty of the object recognition task in this condition, leaving less resources available to devote to encoding the background information. These findings provide important insight into the role of object recognition and task demand during encoding in the BE phenomenon which will be important for current and future models.

\section{References}

Bramao, I., Faisca, L., Petersson, K. M., \& Reis, A. (2010). The influence of surface color information and color knowledge information in object recognition. American Journal of Psychology, 123(4), 437446.

Hale, R. G., Brown, J. M., McDunn, B. A., \& Siddiqui, A. P. (2014). An influence of extremal edges on boundary extension. Psychonomic Bulletin \& Review, 22(4), 961-966. doi:10.3758/s13423-014-0751$\mathrm{X}$

Intraub, H. (2010). Rethinking scene perception: A multisource model. Psychology of Learning and Motivation, 52, 231-264.

Intraub, H. (2012). Rethinking visual scene perception. Wiley Interdisciplinary Reviews: Cognitive Science, 3(1), 117-127.

Intraub, H., Daniels, K. K., Horowitz, T. S., \& Wolfe, J. M. (2008). Looking at scenes while searching for numbers: Dividing attention multiplies space. Perception \& Psychophysics, 70, 1337-1349.

Intraub, H., \& Richardson, M. (1989). Wide-angle memories of close-up scenes. Journal of Experimental Psychology: Learning, Memory, and Cognition, 15(2), 179-187.

McDunn, B. A., Siddiqui, A. P., \& Brown, J. M. (2014). Seeking the boundary of boundary extension. Psychonomic Bulletin \& Review. doi:10.3758/s13423-013-0494-0

Nagai, J., \& Yokosawa, K. (2003). What regulates the surface color effect in object recognition: color diagnosticity or category? Technical Report on Attention and Cognition, 28, 1-4.

Tanaka, J., \& Presnell, L. (1999). Color diagnosticity in object recognition. Perception \& Psychophysics, 61, 1140-1153.

Tanaka, J., Weiskopf, D., \& Williams, P. (2001). The role of color in highlevel vision. Trends in Cognitive Sciences, 5, 211-215.

Van Belle, G., Graef, P. D., Verfaillie, K., Rossion, B., \& Lefevre, P. (2010). Face inversion impairs holilstic perception: Evidence from gaze-contingent stimulation. Journal of Vision, 10(5), 1-13. 